\title{
SEMICONDUTTORI NANOSTRUTTURATI PER APPLICAZIONI TERMOELETTRICHE: UN CONCETTO INNOVATIVO DIMOSTRATO AL COMPUTER
}

\author{
LUCIANO COLOMBO (*) \\ Nota presentata dal m.e. Giorgio Benedek \\ (Adunanza del 13 marzo 2014)
}

SUNTO. - In questa Nota discuterò le proprietà di trasporto termico in materiali nanostrutturati a base di lega silicio-germanio per applicazioni termoelettriche. Dimostrerò, tramite l'uso di simulazioni al computer, che è possibile progettare un nanocomposito con proprietà termiche tali da ottimizzare la figura di merito termoelettrica. In particolare, gli esperimenti computazionali qui discussi dimostreranno in modo quantitativo che il flusso di fononi (i mediatori microscopici del trasporto di calore) dalle regioni calde alle regioni fredde è ostacolato in modo controllabile dai bordi di grano presenti nel nanocomposito. Utilizzando la loro densità come parametro di ingegnerizzazione è possibile progettare il nanocomposito con le prestazioni ottimali.

$$
* * *
$$

ABSTRACT. - I will discuss the thermal transport properties of nanostructured silicongermanium alloys for thermoelectric applications. By means of atomistic simulations, I will show that it is in fact possible to design a nanocomposite with optimal thermoelectric performances, i.e. maximizing its figure of merit. In particular, computer experiments provide quantitative evidence that the phonon flux from hot to cold regions (phonons are the microscopic heat carriers) is dramatically affected by the network of grain boundaries present in the nanocomposite.

(*) Dipartimento di Fisica, Università di Cagliari, Cittadella Universitaria, 09042 Monserrato (CA), Italy.

E-mail: luciano.colombo@unica.it 


\section{INTRODUZIONE E MOTIVAZIONI}

La nostra moderna società industrializzata è estremamente energivora, come ampiamente documentato in un recente rapporto pubblicato dalla Società Italiana di Fisica [1] e come anche riconosciuto dalla Unione Europea, che ha posto questo problema al centro del proprio programma di ricerca e sviluppo tecnologico "Horizon 2020". La necessità di soddisfare il crescente fabbisogno (e consumo) energetico rappresenta, pertanto, una delle più importante sfide poste alla comunità scientifica internazionale.

Da diversi anni la risposta si sta articolando in un massiccio sforzo di ricerca fondamentale (studio di nuovi materiali) e applicativa (sviluppo di nuove tecnologie di produzione energetica ad elevata efficienza e ridotto impatto ambientale). Nell'ambito di questo ampio panorama e da esso motivati, risulta utile condurre anche ricerche di carattere teorico-computazionale volte alla progettazione di nuovi materiali che, manipolati opportunamente alla scala spaziale dei loro costituenti fondamentali, offrano proprietà fisiche non riscontrabili in materiali naturali e tali da garantirne un efficiente sfruttamento in campo energetico.

In questa Nota discuterò, in particolare, il fenomeno del trasporto termico in leghe nanostrutturate a base silicio-germanio che, a mio avviso, rappresenta non solo un interessante problema di fisica dei materiali, ma offre anche la concreta prospettiva di una sua applicazione nel campo della produzione di energia tramite conversione termoelettrica.

Usando lo strumento delle simulazioni al computer, dimostrerò come un concetto teorico possa essere validato dalla simulazione, compreso nei suoi fondamenti microscopici e, quindi, reso concretamente operativo per le applicazioni di interesse.

\section{GENERALITÀ SUI MATERIALI TERMOELETTRICI}

I termoelettrici sono materiali capaci di convertire calore (o, meglio: gradienti di temperatura) in corrente elettrica [2]. L'efficienza di conversione termoelettrica ( $\mathrm{ad}$ una data temperatura $\mathrm{T})$ è riassunta dalla figura di merito $\mathrm{ZT}=\left(\mathrm{S}^{2} \sigma / \kappa\right) \mathrm{T}$ (dove $\mathrm{S}$ è il coefficiente Seebeck, $\sigma$ la conducibilità elettrica e $\kappa$ la conducibilità termica). Essa definisce le proprietà fisiche intrinseche che deve possedere il materiale termoelettrico ideale: è necessario che esso sia un buon conduttore di elettricità, ma che sia al contempo anche un buon isolante termico [3]. 
Questa condizione, purtroppo, non è facilmente riscontrabile nei materiali naturali, stante la legge di Wiedemann-Franz che stabilisce che il rapporto $\kappa / \sigma$ (a temperatura assegnata $T$ ) sia costante. Dunque: un buon conduttore di elettricità è anche un buon conduttore di calore, come dimostrato sperimentalmente dai metalli (è vero anche il contrario: in generale, un buon isolante termico è anche un buon isolante elettrico). Ne segue che, nella maggior parte dei materiali naturali, la figura di merito sia piccola e, dunque, sia bassa l'efficienza complessiva della trasformazione di calore in elettricità.

Questo risultato di fisica fondamentale è alla base dal limitato impiego pratico che sin qui hanno avuto i termoelettrici. Sebbene la fisica del processo sia nota da circa due secoli [4], le sue applicazioni tecnologiche sono ancora limitate a mercati di nicchia (tipicamente, quelli areonautico e spaziale), dove bassa efficienza, alti costi e limitata disponibilità dei materiali naturali sono fattori limitanti meno importanti della affidabilità e durata temporale del dispositivo termoelettrico con essi costruito [5]. Ove fossero, invece, disponibili nuovi materiali ad alta ZT si potrebbe immaginarne un uso a larga scala come, ad esempio, nell'industria automobilistica: sfruttando il calore estratto dai gas di scarico accumulati nella marmitta, si potrebbe generare gran parte della corrente necessaria ad alimentare tutte le servitù elettriche presenti nell'automezzo, con un significativo risparmio energetico. Lo stesso principio, poi, potrebbe essere esteso all'industria pesante (tipicamente, quella siderurgica) dove il calore risultante dal processo produttivo viene normalmente dissipato nell'ambiente, senza sfruttamento utile.

Il punto chiave, dunque, è quello di massimizzare ZT, ovvero: ridurre $\kappa$, pur mantenendo $\sigma$ a valori opportunamente alti. Di recente [6], nell'ambito del paradigma materials-by-design (progettazione di nuovi materiali a partire dalla struttura atomistica, con proprietà fisiche ottimali per una certa applicazione) è stata proposto un nuovo concetto molto promettente che prevede di introdurre in un solido buon conduttore di corrente alcune caratteristiche strutturali artificiali tali da ridurne significativamente la capacità di sostenere un flusso termico (senza però alterare quello elettrico) [6].

Il concetto si basa sulla natura microscopica dei fenomeni di trasporto di corrente e calore, nonché sull'effetto che i difetti strutturali nel reticolo atomico che forma il solido hanno sui due fenomeni. Nel primo caso si tratta di un flusso di elettroni, accelerati da una differenza di potenziale elettrico; nel secondo caso si tratta di un flusso di pseudo- 
particelle, dette fononi, descriventi il complesso dei moti di agitazione termica di un reticolo atomico [7] il cui flusso si instaura dalle regioni più calde verso quelle più fredde. Il moto di elettroni e fononi (rispettivamente, sotto l'azione di gradienti elettrici e termici) è ostacolato dalle interazioni mutue e da quelle con eventuali difetti strutturali presenti nel materiale. Si definisce libero cammino medio lo spazio in media percorso da un elettrone o da un fonone tra due eventi di interazione consecutivi: maggiore sarà tale cammino, maggiormente intensa risulterà la corrispondente corrente. Queste interazioni danno origine, dunque, ai fenomeni di resistenza elettrica e termica.

Acquisite queste nozioni, possiamo riassumere il nuovo concetto materials-by-design come segue: per aumentare il valore di ZT in un materiale si devono introdurre difetti strutturali tali da aumentare notevolmente la resistenza termica, senza alterare significativamente il flusso degli elettroni. Ciò può essere fatto introducendo ostacoli reticolari che, per loro distribuzione spaziale, siano distanziati in modo da interferire, riducendolo, con il libero cammino medio dei fononi (normalmente molto più grande della tipica distanza tra atomi in un reticolo), ma non con quello degli elettroni (il cui libero cammino medio è, normalmente, molto inferiore a quello dei fononi). Riassumendo: il problema è riformulato in termini di creazione di una distribuzione di difetti reticolari capaci di ridurre efficacemente il libero cammino medio fononico, lasciando quello elettronico praticamente inalterato.

\section{I NANOCOMPOSITI SILICIO-GERMANIO}

Si prestano ad implementare in pratica il concetto discusso nel precedente paragrafo i materiali semiconduttori, nella specie delle leghe silicio-germanio (un reticolo cristallino tipo zincoblenda, i cui siti sono occupati a caso da atomi delle due specie, in proporzione variabile e controllabile).

In tali materiali, infatti, la conduzione di elettricità è perfettamente sotto controllo, tramite il metodo del drogaggio chimico mutuato dalla microelettronica: in sostanza, possiamo generare una lega a stechiometria e drogaggio assegnati, tali da avere il valore di $\sigma$ utile per l'applicazione termoelettrica di interesse. Al contempo, però, essi possono facilmente essere sottoposti a trattamenti di routine (tipicamente, micro-macinazione e successivo ricompattamento meccanico) che li rendono in forma 
finale nanocristallina. In altre parole, a livello atomistico tali strutture consistono in una distribuzione di grani cristallini (quindi, a struttura reticolare ordinata) di dimensione nanometrica e orientati a caso nello spazio, interfacciati l'uno all'altro tramite superfici di contatto dette "bordi di grano". Un esempio è rappresentato in Fig. 1 dove viene visualizzata la struttura atomistica di tre diversi nanocompositi che differiscono per granulometria, avvero per il diverso valore $d_{a v e}$ della dimensione media dei grani cristallini. Orbene, la griglia dei bordi di grano genera una altissima resistenza termica, che si traduce in una notevole riduzione della conducibilità $\kappa$. Al contempo, però, predeterminando il valore $d_{a v e}$ in modo opportuno è possibile selezionare un nanocomposito le cui dimensioni di grano siano maggiori del libero cammino medio elettronico, lasciando così immutata $\sigma$ al valore della lega silicio-germanio definita dalla specifica stechiometria assegnata. Insomma: è possibile creare un "isolante fononico" che sia però un buon "conduttore elettronico", superando i limiti imposti dalla legge di Wiedemann-Franz.
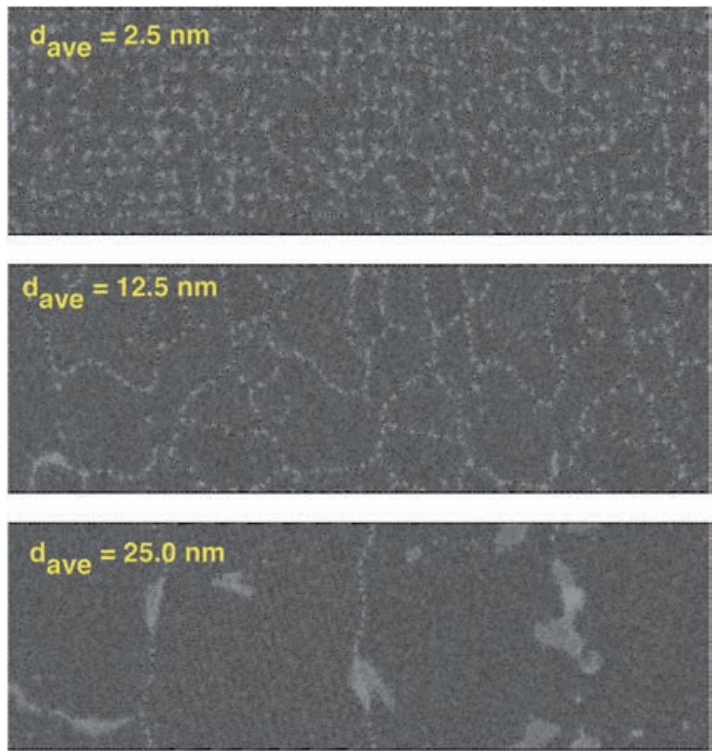

Fig. 1 - Rappresentazione della struttura di tre diversi nanocompositi di lega silicio-germanio, a differente granulometria (descritta dal parametro $\mathrm{d}_{\text {ave }}$ ), cosi come ottenuta da simulazioni al computer. La rete dei bordi di grano risulta in contrasto più chiaro e identifica le zone con alto disordine reticolare che contribuiscono maggiormente a limitare il libero cammino medio dei fononi. Esse agiscono come sorgente di resistenza termica. 
I primi esperimenti di laboratorio [8] hanno già dimostrato la bontà di questo concetto innovativo, ma hanno altresì lasciato aperte numerose questioni di carattere fondamentale, tra cui: (i) la determinazione di quale sia la migliore combinazione di stechiometria e granulometria che minimizzi in modo assoluto il valore di conducibilità $\kappa$ e (ii) l'elucidazione del ruolo dei bordi di grano nell'ostacolare il flusso di fononi attraverso la struttura.

Per dare risposta a questi interrogativi, è stata progettata e realizzata una complessa ed onerosa campagna di simulazioni al computer, descritta dettagliatamente nel seguito, dove il fenomeno della conduzione termica in nanocompositi silicio-germanio è stato accuratamente studiato a livello atomistico.

\section{LE SIMULAZIONI DI DINAMICA MOLECOLARE}

La dinamica molecolare (DM) è un metodo simulativo generale ed affidabile, capace di fornire predizioni quantitative sulle proprietà fisiche di un un materiale in qualsivoglia stato di aggregazione, composizione chimica e grado di ordine strutturale. In breve, la DM consiste nella soluzione numerica (cioè eseguita su computer) delle equazioni del moto di un insieme di atomi interagenti sotto l'azione di arbitrari vincoli termodinamici (cioè, in condizioni di temperatura, pressione, densità, ... assegnate). Una volta fornito alla DM uno schema di interazione tra atomi (effettivamente dispobile per lo specifico sistema silicio-germanio di nostro interese), è possibile calcolare le loro traiettorie spaziotemporali, in modo da esplorare tutta la porzione dello spazio delle fasi accessibile al sistema simulato sotto $i$ vincoli termodinamici assegnati [9]. Pertanto, questo dispositivo teorico-computazionale è in grado di calcolare una qualunque osservabile fisica come una media di insieme, secondo i dettami della meccanica statistica.

Lo schema DM fornisce, in particolare, un modo diretto e concettualmente semplice per il calcolo della conducibilità termica quando il sistema viene inizialmente preparato in una condizione di non equilibrio e, successivamente, ne viene simulata la naturale evoluzione verso l'equilibrio. La situazione è schematicamente riassunta in Fig. 2: un sistema è inizialmente preparato in modo che le sue metà destra e sinistra si trovino a temperatura diversa (ovvero: che il profilo di temperatura lungo la direzione $z$ indicata in figura sia "a gradino"); a questo punto il sistema è 
simulato in condizioni microcanoniche (ovvero: senza che sia imposto alcun vincolo termodinamico, se non la conservazione del numero di particelle, del volume e dell'energia totale) e, pertanto, tenderà ad evolvere spontaneamente verso la condizione di equilibrio, caratterizzata da una temperatura uniforme in tutto il sistema (pari al valore medio delle due temperature iniziali). Seguendo il sistema in questo suo approccio all'equilibrio, è possibile calcolare ad ogni istante di tempo $t$, durante la simulazione DM, la differenza $\Delta \mathrm{T}(\mathrm{t})=\left\langle\mathrm{T}_{\mathrm{s}}\right\rangle-\left\langle\mathrm{T}_{\mathrm{d}}\right\rangle$ tra le due temperature medie $<\mathrm{T}_{\mathrm{s}}>\mathrm{e}<\mathrm{T}_{\mathrm{d}}>$, rispettivamente, delle metà di sinistra e di destra. Si osserva direttamente dai dati estratti dalla simulazione che questa differenza segue un'evoluzione temporale di tipo esponenziale decrescente. Ciò è diretta conseguenza della legge di Fourier: risolvendo l'equazione di diffusione del calore per un profilo iniziale di temperatura del tipo "a gradino" si dimostra infatti che $\Delta T(t)=\Sigma_{n} C_{n} \exp \left[-\alpha_{n} \lambda t\right]$, dove: $\lambda=\kappa / \rho c_{V}$ è la diffusività termica del sistema di cui si assumono noti (o calcolabili) la densità $\rho$ ed il calore specifico (a volume costante) $c_{\mathrm{V}}$. Invece, $\mathrm{i}$ coefficienti $\alpha_{\mathrm{n}}=2 \pi \mathrm{n} / \mathrm{L}_{\mathrm{z}}$ e $\left.\mathrm{C}_{\mathrm{n}}=8\left(<\mathrm{T}_{\mathrm{s}}>-<\mathrm{T}_{\mathrm{d}}>\right) \cos \left(\alpha_{\mathrm{n}} \mathrm{L}_{z} / 2\right)-1\right]^{2} /\left(\alpha_{\mathrm{n}}^{2} \mathrm{~L}_{\mathrm{z}}^{2}\right)$ sono matematicamente imposti dalle condizioni al contorno. Pertanto, la conducibilità termica $\kappa$ è facilmente calcolata dai dati simulativi semplicemente calibrando il valore di $\lambda$ sulla curva temporale $\Delta \mathrm{T}(\mathrm{t})=\left\langle\mathrm{T}_{\mathrm{s}}\right\rangle-\left\langle\mathrm{T}_{\mathrm{d}}\right\rangle$ generata durante la DM.

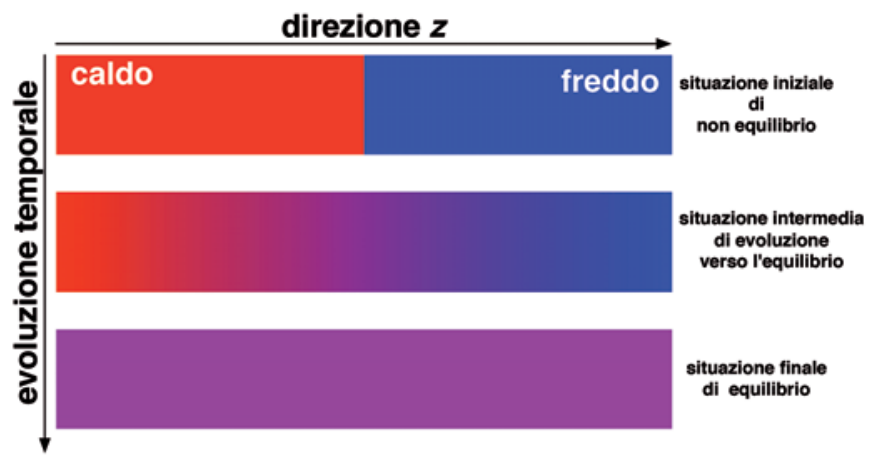

Fig. 2 - Rappresentazione grafica dello schema di simulazione descritto nel testo.

In alto: un sistema si trova inizialmente in una condizione di non equilibrio in cui

le due metà destra e sinistra sono preparate a due temperature differenti.

Al centro: il sistema viene quindi simulato in condizioni microcanoniche e, dunque,

si instaura un flusso di calore (fononi) dalla regione inizialmente più calda a quella inizialmente più fredda.

In basso: al termine della simulazione, il sistema raggiunge una condizione di equilibrio termico caratterizzata da temperatura uniforme. 
Questo schema di simulazione ha numerosi vantaggi pratici [10]. Innanzitutto è molto semplice da implementare e i risultati finali sono sostanzialmente stabili rispetto alla scelta arbitraria delle condizioni di esecuzione della simulazione (per esempio, non dipendono dal reale profilo iniziale di temperatura, nè dai valori di temperatura assegnati alle due metà del sistema). Esso, inoltre, comporta la necessità di seguire il sistema solamente durante il breve transiente temporale di sua evoluzione verso l'equilibrio. Ciò comporta che l'onere computazionale complessivo della simulazione (che dipende linearmente dalla lunghezza temporale della simulazione $\mathrm{DM}$ ) è comparativamente molto ridotto rispetto ad altri metodi pure presenti e discussi in Letteratura. Questa caratteristica, a sua volta, è di fondamentale importanza, perché consente di applicare questa procedura a sistemi di grandi dimensioni (contenenti milioni di particelle), in realtà necessari per descrivere la complessità strutturale di un nanocomposito. Infine, sebbene i risultati sulla fisica del sistema siano estratti da una simulazione numerica, essa ha un robusto fondamento teorico (equazione di diffusione del calore) e, pertanto, posseggono un sicuro valore predittivo.

Nell'ambito dello schema descritto, il protocollo di simulazione adottato è in realtà molto semplice. Una volta realizzato un modello strutturale di nanocomposito con le volute caratteristiche [11], si provvede a prepararlo nella condizioni iniziale della simulazione, come illustrato in Fig. 1. A questo punto, la dinamica microcanonica del sistema è simulata al computer, fino al raggiungimento della condizione finale di equilibrio a temperatura uniforme. Durante questa fase viene calcolato numericamente il termine $\Delta \mathrm{T}(\mathrm{t})=\left\langle\mathrm{T}_{\mathrm{s}}\right\rangle-\left\langle\mathrm{T}_{\mathrm{d}}\right\rangle$ dal quale si estrae, infine, il corrispondente valore di conducibilità termica $\kappa$. La procedura viene quindi ripetuta variando i parametri strutturali (granulometria) e chimici (stechiometria), generando in questo modo un complesso di informazioni che possono essere sottoposte a ulteriore analisi teorica sulla quale basare la nostra comprensione del fenomeno di trasporto termico.

\section{Risultati E LORO Discussione}

Il primo punto importante da affrontare è determinare se, a parità di stechiometria, un nanocomposito abbia effettivamente una conducibilità termica inferiore della corrispondente lega omogenea (ovvero: 
monocristallina, senza bordi di grano). Se così non fosse, il concetto stesso perderebbe di significato e sarebbe inutile sottoporre le leghe ai trattamenti di micro-macinazione e compattazione che, pur se ordinari, comportano comunque oneri di costo.

In Fig. 3 si riportano i valori di $\kappa$, ottenuti per una lega omogenea e per un nanocomposito di granulometria $\mathrm{d}_{\text {ave }}=10.5 \mathrm{~nm}$, al variare della loro stechiometria nell'intervallo di interesse tecnologico. La simulazione fornisce una chiara indicazione quantitativa: per ogni valore di contenuto di germanio nella lega, la condubilità termica del nanocomposito è inferiore di circa il 50\% rispetto a quella della corrispondente lega omogenea. Questo risultato conferma e generalizza quanto già noto sperimentalmente, limitatamente al solo caso di concentrazione di germanio fissa al 20\% [12,13] e dimostra in maniera inequivocabile che la figura di merito termoelettrica di un nanocomposito è circa doppia rispetto a quella della corrispondente lega.

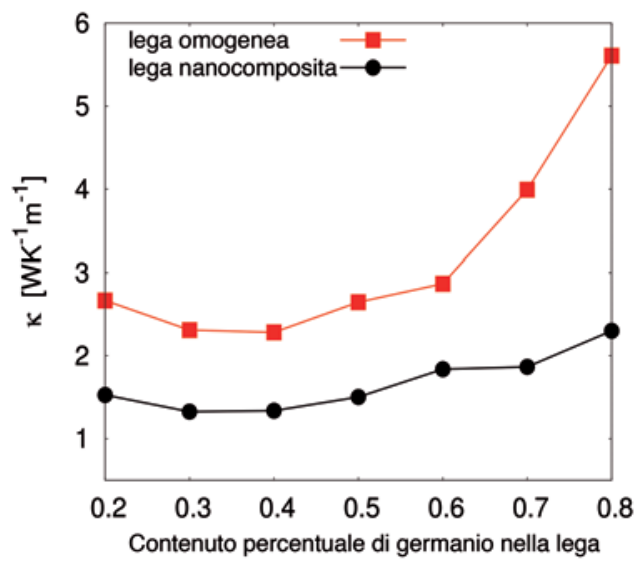

Fig. 3 - Confronto tra i valori calcolati tramite simulazione al computer della conducibilità termica $\kappa$ in leghe omogenee e nanocomposite, al variare del contenuto percentuale di germanio.

Poichè questo risultato è valido ad ogni stechiometria, possiamo concludere che la rete di bordi di grano nel nanocomposito gioca un ruolo fondamentale nel determinare quanto osservato. Quest'affermazione è resa quantitativa tramite il calcolo della funzione di accumulazione termica $\kappa\left(\mathrm{L}_{z}\right) / \kappa(\infty)$, definita dal rapporto tra $\mathrm{i}$ valori di conducibilità calcolati per un nanocomposito di lunghezza $L_{z}$ ed uno 
di lunghezza infinita. In pratica, la funzione di accumulazione termica fornisce la stima quantitativa del contributo alla conducibilità totale esclusivamente da parte dei fononi il cui libero cammino medio è inferiore (o, al più, uguale) ad $\mathrm{L}_{z}$. In Fig. 4 si dimostra che in una tipica lega nanocomposita al $50 \%$ di germanio circa $\sim 80 \%$ della conducibilità è dovuta a fononi con libero cammino medio inferiore ai $100 \mathrm{~nm}$. D'altro canto in una lega omogenea di identica stechiometria lo stesso contributo è fornito da fononi con libero cammino medio fino a $\sim 1 \mu \mathrm{m}$. È, dunque, del tutto evidente che i difetti reticolari tipo "bordo di grano" generano un importante fenomeno di resistenza termica: essi, infatti, troncano la lunghezza di diffusione dei portatori microscopici del calore (i fononi) ad un valore inferiore di circa un ordine di grandezza.

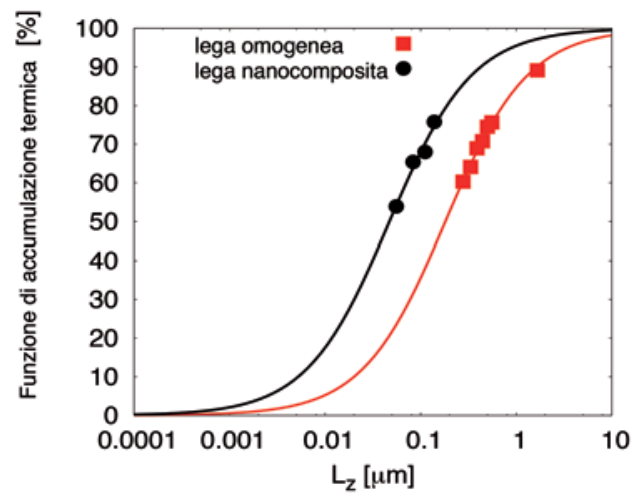

Fig. 4 - Funzione di accumulazione termica per una lega omogenea o nanostrutturata al $50 \%$ di contenuto di germanio.

Al fine di caratterizzare ancor meglio il ruolo della nanostruttura sulle proprietà di trasporto termico, possiamo studiare gli effetti della granulometria con un nuovo esperimento di alchimia computazionale. A tal fine, fissiamo la stechiometria al valore $50 \%$ di germanio e studiamo come varia la conducibilità al variare del parametro $\mathrm{d}_{\text {ave }}$. I risultati sono riassunti in Fig. 5: la conducibilità aumenta in modo monotono con l'aumentare di $\mathrm{d}_{\text {ave }}$ e, per valori sufficientemente grandi, si ritrova il valore di conducibilità proprio della lega omogenea (di identica stechiometria). In conclusione, la simulazione chiaramente dimostra che è inutile l'impiego di nanocompositi a granulometria troppo grande perché la densità della corrispondente rete di bordi di grano è troppo bassa per assicurare un efficiente fenomeno di resistenza termica. 
Un altro risultato interessante dalla simulazione è che la conducibilità termica è ridotta ai minimi valori per $\mathrm{d}_{\text {ave }} \leq 15 \mathrm{~nm}$. In particolare, stante quanto riportato in Fig. 5, il minimo assoluto di $\kappa$ per un nanocomposito silicio-germanio è di $\sim 1 \mathrm{WK}^{-1} \mathrm{~m}^{-1}$, ottenuto per una granulometria di circa 2-3 nm. Questo valore è ben 3-4 volte inferiore a quello di lega, il che si riflette in un significativo aumento della figura di merito termoelettrica. Al di sotto questo valore non si può scendere, per motivi termodinamici: per dimensioni inferiori, infatti, i grani si trovano sotto la soglia di capillarità [14] e, dunque, tendono ad evaporare se lasciati a temperature sufficientemente alte, come in effetti accadrebbe in un dispositivo termoelettrico che normalmente funziona a molte centinaia di gradi (tra i 400 e i 800 gradi Kelivin). Questa predizione, dunque, può essere molto utile in pratica, al fine di definire le caratteristiche ottimali del materiale di impiego in un eventuale dispositivo.

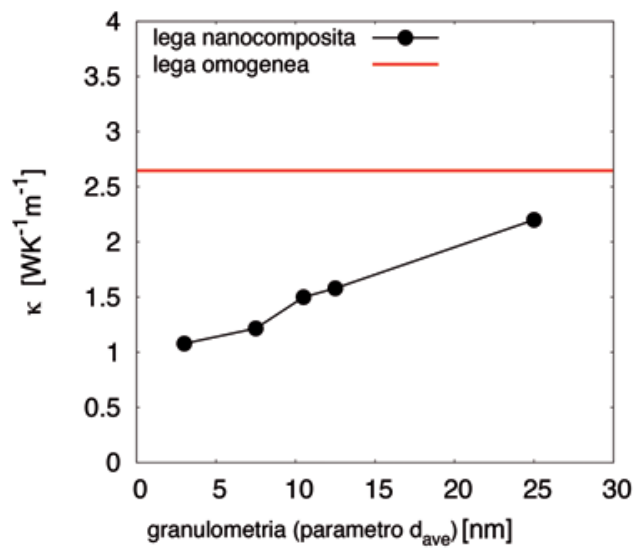

Fig. 5 - Valori di conducibilità termica in una lega nanocomposita (al 50\% di contenuto di germanio) calcolati al variare della sua granulometria.

\section{ConClusioni}

In questa breve Nota ho sviluppato tre concetti in parallelo: (i) la simulazione al computer è in grado di fornire predizioni quantitative precise ed affidabili sulle proprietà fisiche di (nuovi) materiali per applicazioni tecnologiche avanzate e, pertanto, può guidare (ii) un paradigma materials-by-design tramite il quale, manipolando la materia alla scala dei suoi costituenti elementari, è possibile progettare nuovi 
materiali nanostrutturati con proprietà non altrimenti riscontrate in Natura.

La combinazione di questi due concetti ha portato alla capacità di (iii) predire le condizioni ottimali di composizione chimica e nanostruttura in leghe silicio-germanio per un loro efficiente impiego come energy materials e, in particolare, per una efficiente conversione termoelettrica.

\section{BIBLIOGRAFIA}

[1] Società Italiana di Fisica (2008), "Energy in Italy: problems and perspectives (1999-2020)", documento disponibile sul sito: http://www.sif.it/attivita/energy/ librobianco

[2] G.S. Nolas, J. Sharp, and H. Goldsmid, "Thermoelectrics: Basic Principles and New Materials Developments" (Springer, New York, 2001).

[3] D.K.C. McDonald, "Thermoelectricity" (Dover, New York, 2006).

[4] L. Anatychuk, J. Stockholm, G. Pastorino, "On the discovery of thermoelectricity by A. Volta", Proceedings of the $8^{\text {th }}$ European Conference on Thermoelectrics (2010), pp. 10-15.

[5] G.J. Snyder and E.B. Toberer, "Complex thermoelectric materials", Nature Materials (2008) vol. 7, pp. 105 - DOI:10.1038/nmat2090.

[6] M. Dresselhaus, G. Chen, M.Y. Tang, R.G. Yang, H. Lee, D.Z. Wang, Z.F. Ren, J.-P. Fleurial and P. Gogna, "New directions in low-dimensional thermoelectric materials", Advanced Materials (2007) vol. 19, pp. 1043 - DOI:10.1002/adma. 200600527.

[7] N.W. Ashcroft, N.D. Mermin, "Solid state physics" (Saunders College Publishing, Orlando, 1976).

[8] M. Zebarrjadi, K. Esfarjani, M.S. Dresselhaus, Z.F. Ren, and G. Chen, "Perspectives on thermoelectrics: from fundamentals to device applications", Energy \& Environmental Science (2012), vol. 5, pp. 5147 - DOI:10.1039/ C1EE02497C.

[9] D. Frenkel and B. Smit, "Understanding molecular simulation" (Academic Press, San Diego, 1996).

[10] C. Melis, R. Dettori, S. Vandermeulen, L. Colombo, "Calculating lattice thermal conductivity in a transient conduction regime: theory and implementation", Eur. Phys. J. B (2014), vol. 87, pp. 96 - DOI:10.1140/epjb/e2014-50119-0.

[11] C. Melis, L. Colombo, "Lattice thermal conductivity in $S i_{x} G e_{1-x}$ nanocomposites" (2014), Physical Review Letters vol. 112, pp. 065901 - DOI:10.1103/ PhysRevLett.112.065901.

[12] X.W. Wang, H. Lee, Y.C. Lan, G.H. Zhu, G. Joshi, D.Z. Wang, J. Yang, A.J. Muto, M.Y. Tang, J. Klatsky, S. Song, M. S. Dresselhaus, G. Chen, and Z. F. Ren, "Enhanced thermoelectric figure of merit in nanostructured n-type silicon germa- 
nium bulk alloy”, Applied Physics Letters (2008), vol. 93, pp. 193121 - DOI: 10.1063/1.3027060.

[13] G. Joshi, H. Lee, Y. Lan, X. Wang, G. Zhu, D. Wang, R.W. Gould, D.C. Cuff, M.Y. Tang, M.S. Dresselhaus, G. Chen, Z. Ren, "Enhanced Thermoelectric Figure-of-Merit in Nanostructured p-type Silicon Germanium Bulk Alloys", Nano Lett. (2008), vol. 8, pp. 4670 - DOI:10.1021/nl8026795.

[14] A. Mattoni, L. Colombo, "Crystallization kinetics of mixed amoprhous-crystalline nanosystems”, Physical Review B (2008), vol. 78, pp. 075408 - DOI:10.1103/ PhysRevB.78.075408. 